Supporting Information:

\title{
Highly similar sequence and structure yet different biophysical behaviour: A computational study of two triosephosphate isomerases
}

Cecilia Chávez-García ${ }^{1,2}$ and Mikko Karttunen ${ }^{1,2,3}$

${ }^{1}$ Department of Chemistry, the University of Western Ontario, 1151 Richmond Street, London, Ontario N6A 5B7, Canada

${ }^{2}$ The Centre of Advanced Materials and Biomaterials Research, the University of Western Ontario, 1151 Richmond Street, London, Ontario, N6A 5B7, Canada

${ }^{3}$ Department of Physics and Astronomy, the University of Western Ontario, 1151 Richmond Street, London, Ontario, N6A 3K7, Canada 

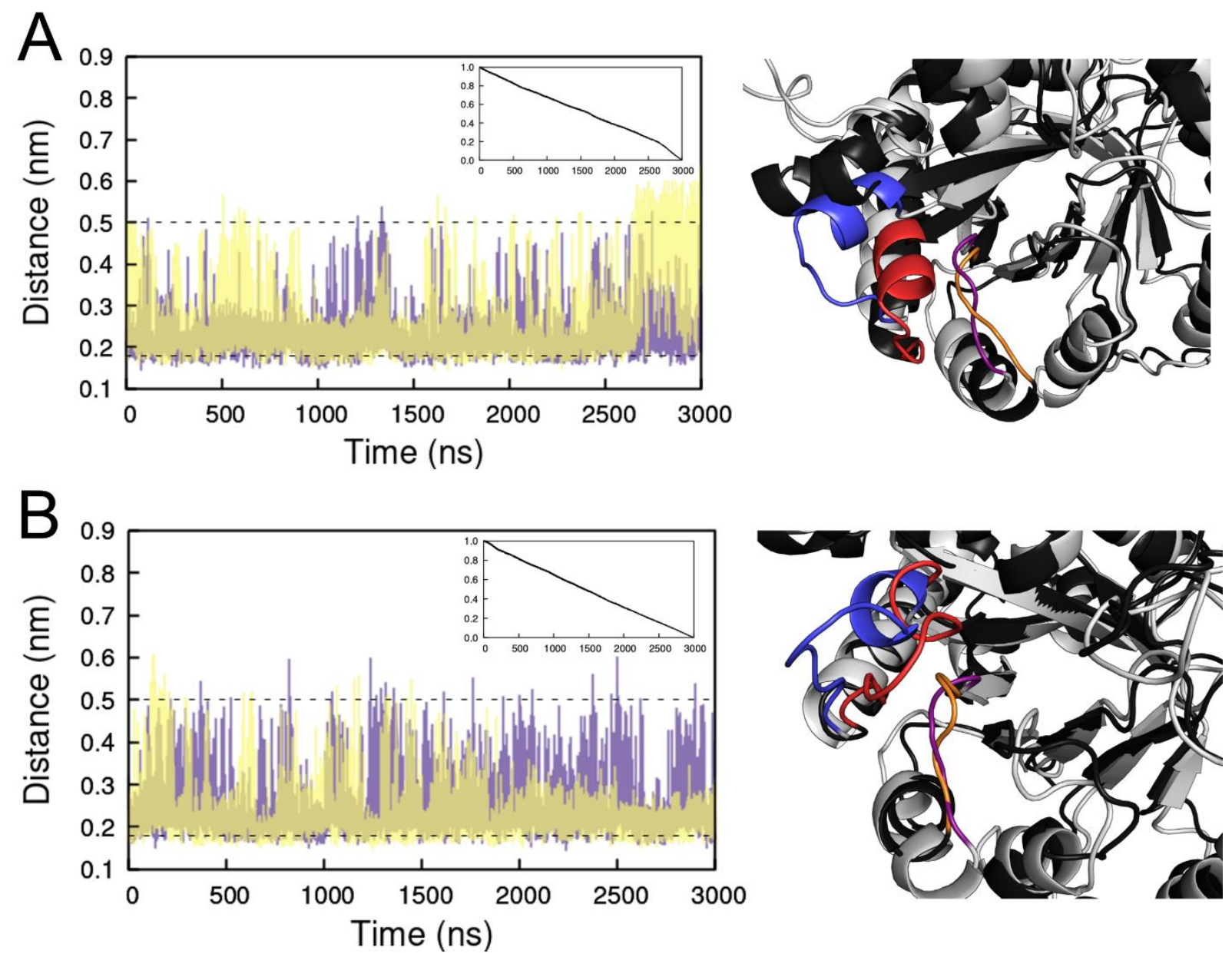

Fig. S1. Minimum distance between loops 6 and 7 in TbTIM (A) and Mut1 (B) for monomer A (purple) and monomer B (yellow). Dashed lines mark the two different states sampled by the loops. Inside: crosscorrelation between the loop state of the two monomers. Right: alignment of the open (gray) and closed (black) conformations. Loop 6 is shown in red for the closed conformation and blue for the open conformation, loop 7 is shown in orange (closed) and purple (open). 

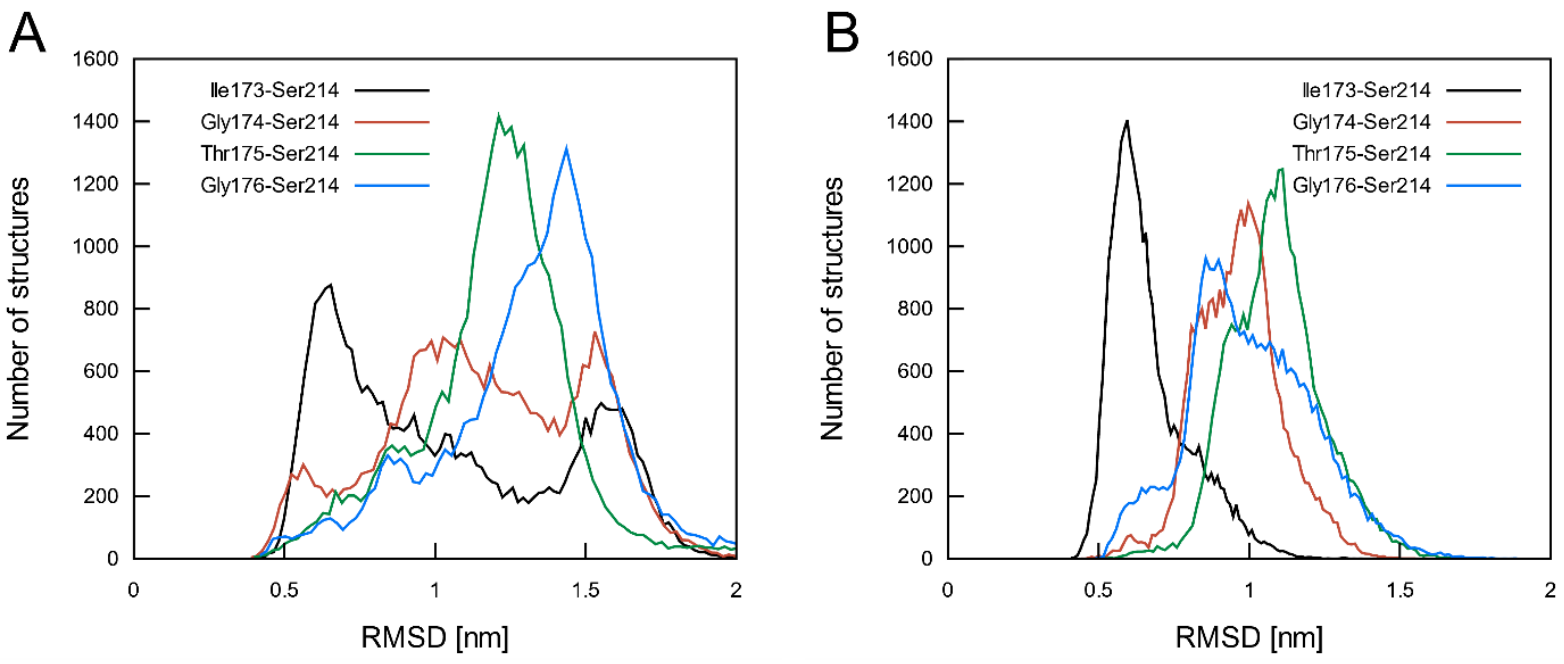

Fig. S2. Monitoring the movement of loop 6 during our TcTIM simulation in (A) monomer A and (B) monomer B. The loop 6 conformation (open and close) was defined as a measure of the distances between the C $\alpha$ atoms of residues Ile173, Gly172, Thr175 and Gly176 on loop 6 and the C $\alpha$ atom of Ser214 in loop 7. Loop 6 samples both open and close conformations in monomer A, however, prefers to remain in a closed state in monomer B.
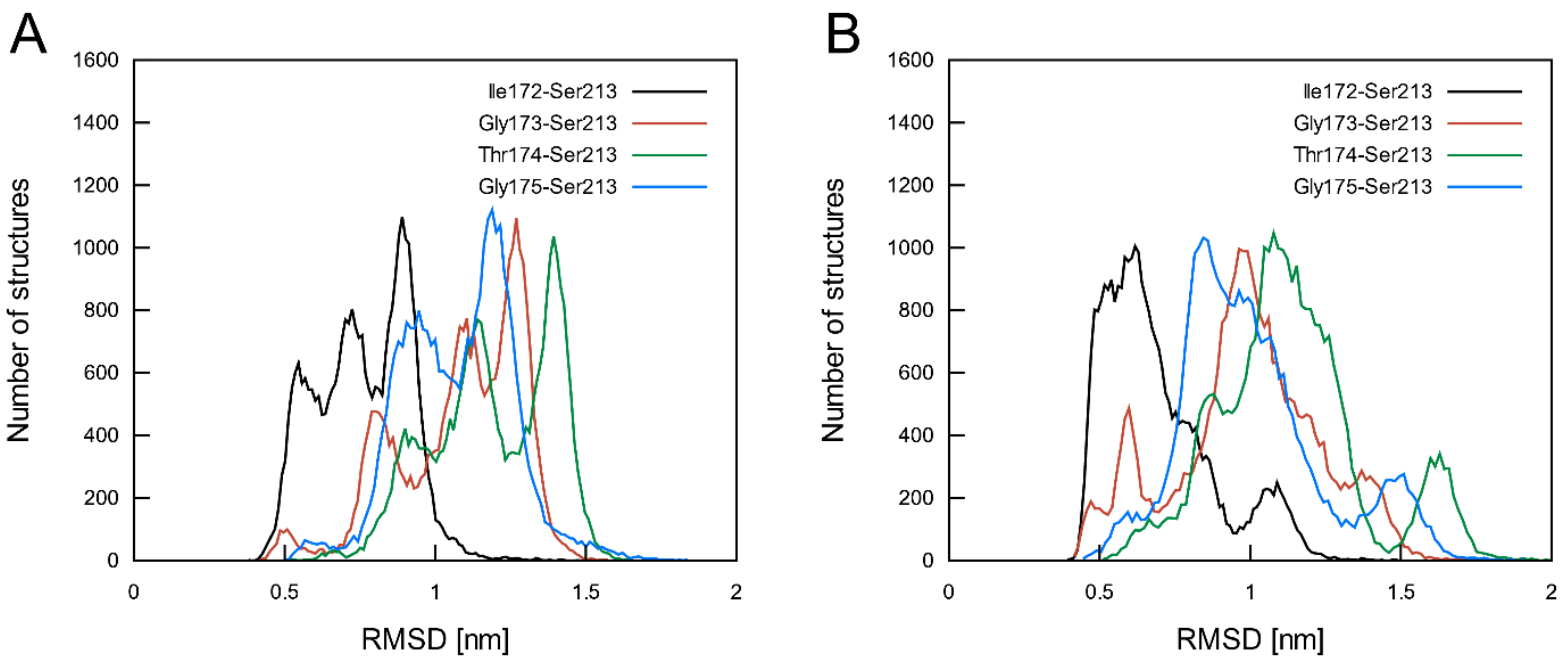

Fig. S3. Monitoring the movement of loop 6 during our TbTIM simulation in (A) monomer A and (B) monomer B. The loop 6 conformation (open and close) was defined as a measure of the distances between the C $\alpha$ atoms of residues Ile173, Gly172, Thr175 and Gly176 on loop 6 and the C $\alpha$ atom of Ser214 in loop 7. Loop 6 samples preferentially the closed conformation in both monomers, sampling transiently an open conformation in monomer B. 

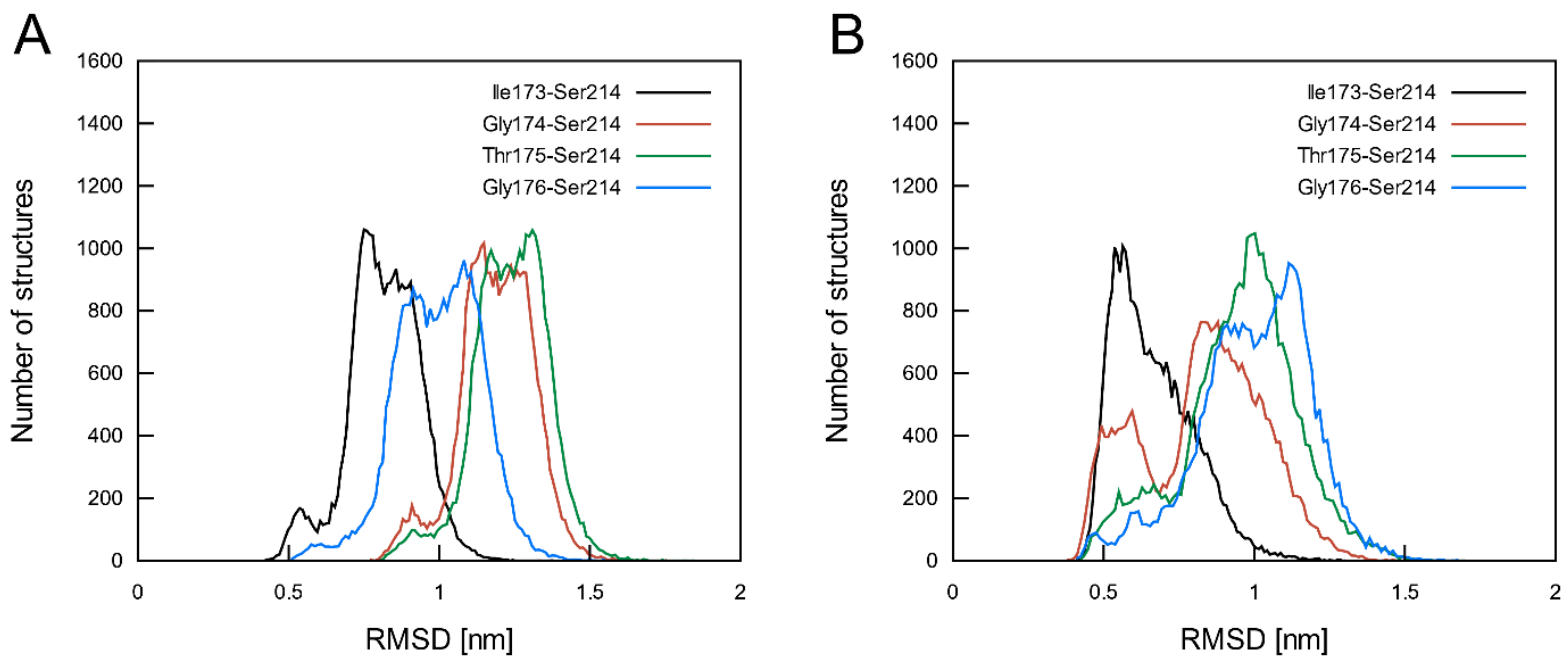

Fig. S4. Monitoring the movement of loop 6 during our Mut1 simulation in (A) monomer A and (B) monomer B. The loop 6 conformation (open and close) was defined as a measure of the distances between the $\mathrm{C} \alpha$ atoms of residues Ile173, Gly172, Thr175 and Gly176 on loop 6 and the C $\alpha$ atom of Ser214 in loop 7. Loop 6 remains in an intermediate state in monomer A, where it is not fully closed or fully opened, and samples a closed conformation in monomer B.
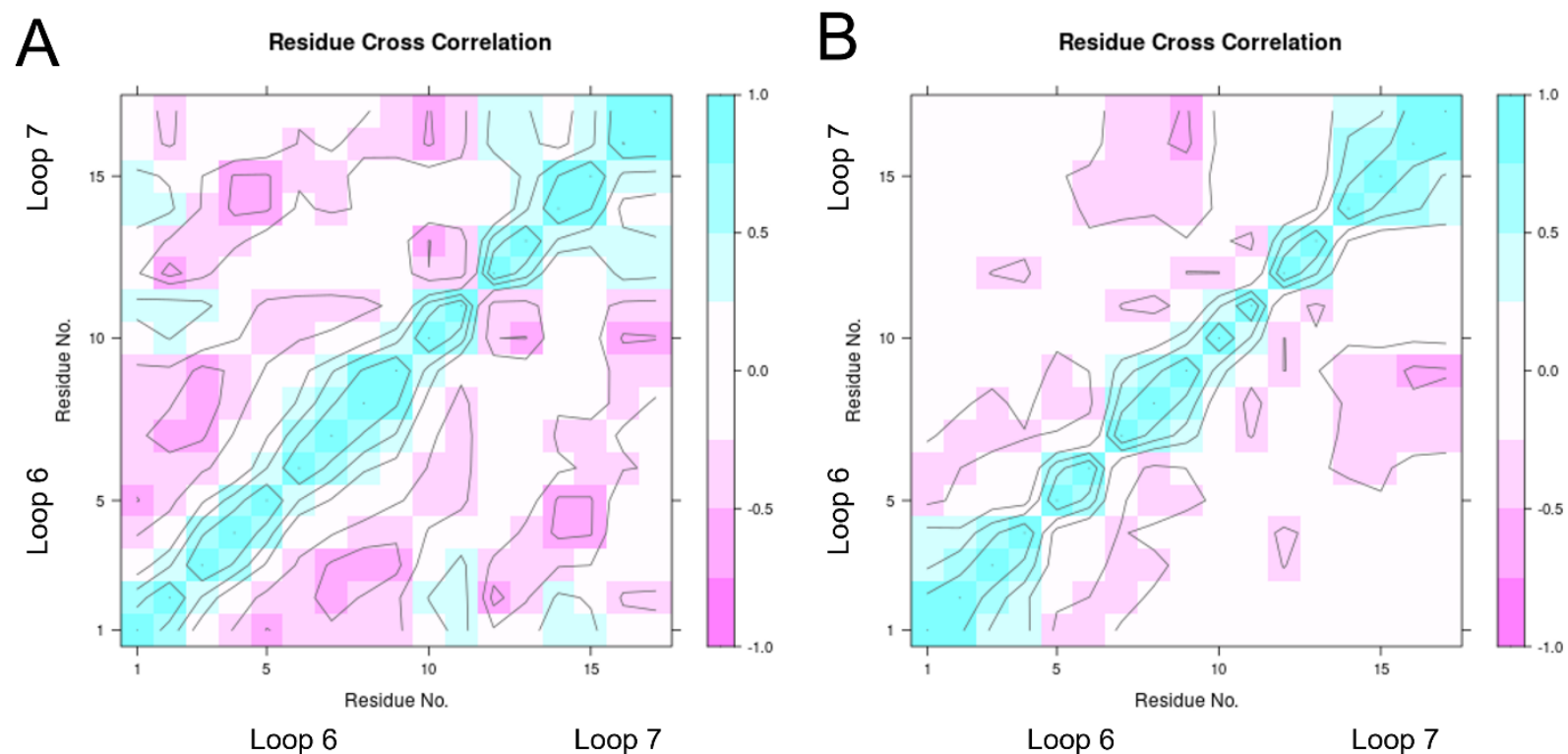

Fig. S5. Cross correlation between $\mathrm{C} \alpha$ atoms in loops 6 and 7 in the TcTIM simulation in: (A) monomer A and (B) monomer B. Loop 6 corresponds to the first 11 amino acids and loop 7 to residues 12 to 17 . The anti-correlation observed between the loops is in good agreement with the open-close transition observed in them. 

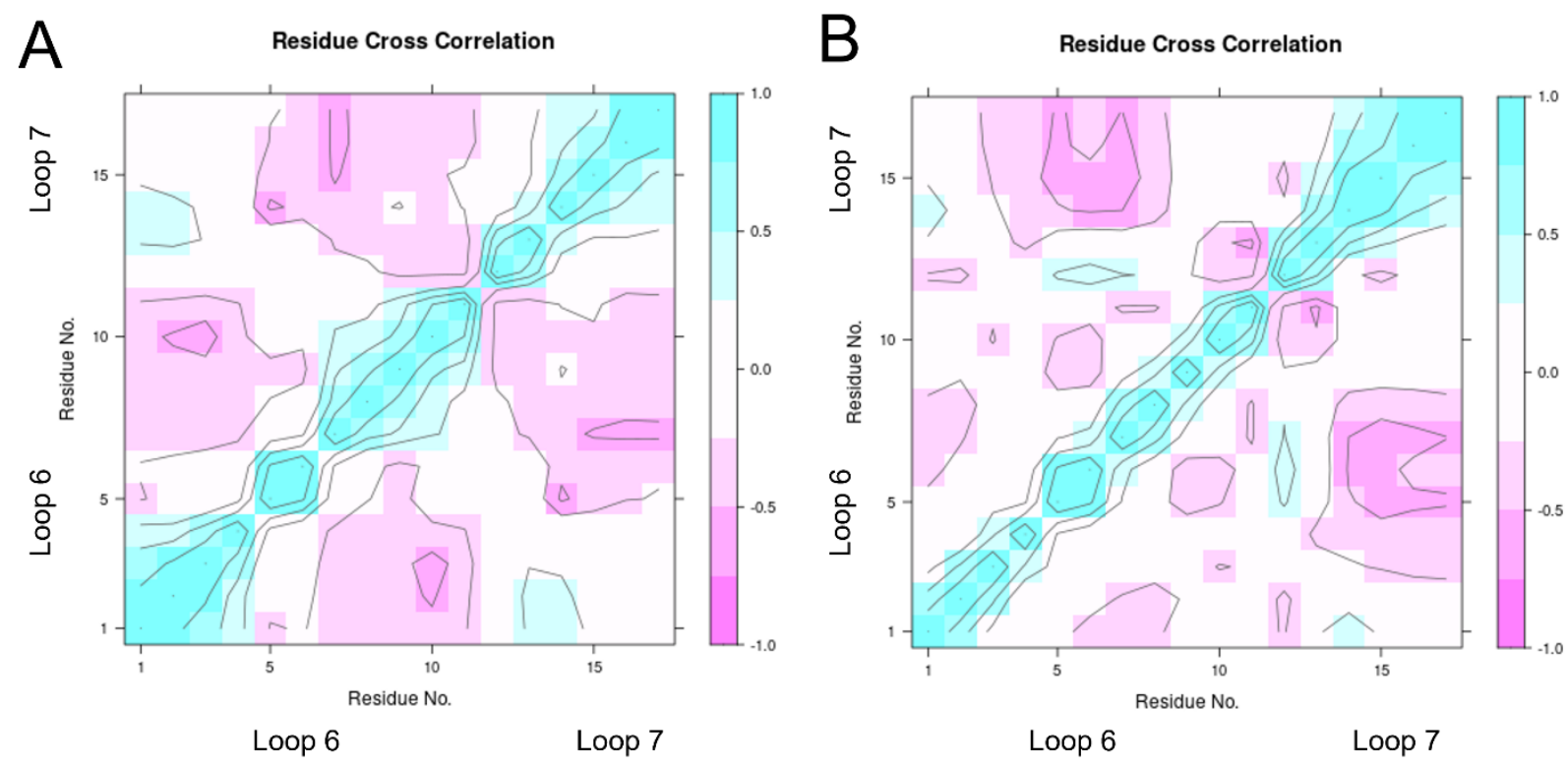

Fig. S6. Cross correlation between $\mathrm{C} \alpha$ atoms in loops 6 and 7 in the TbTIM simulation in: (A) monomer A and (B) monomer B. Loop 6 corresponds to the first 11 amino acids and loop 7 to residues 12 to 17 . The anti-correlation observed between the loops is in good agreement with the open-close transition observed in them.
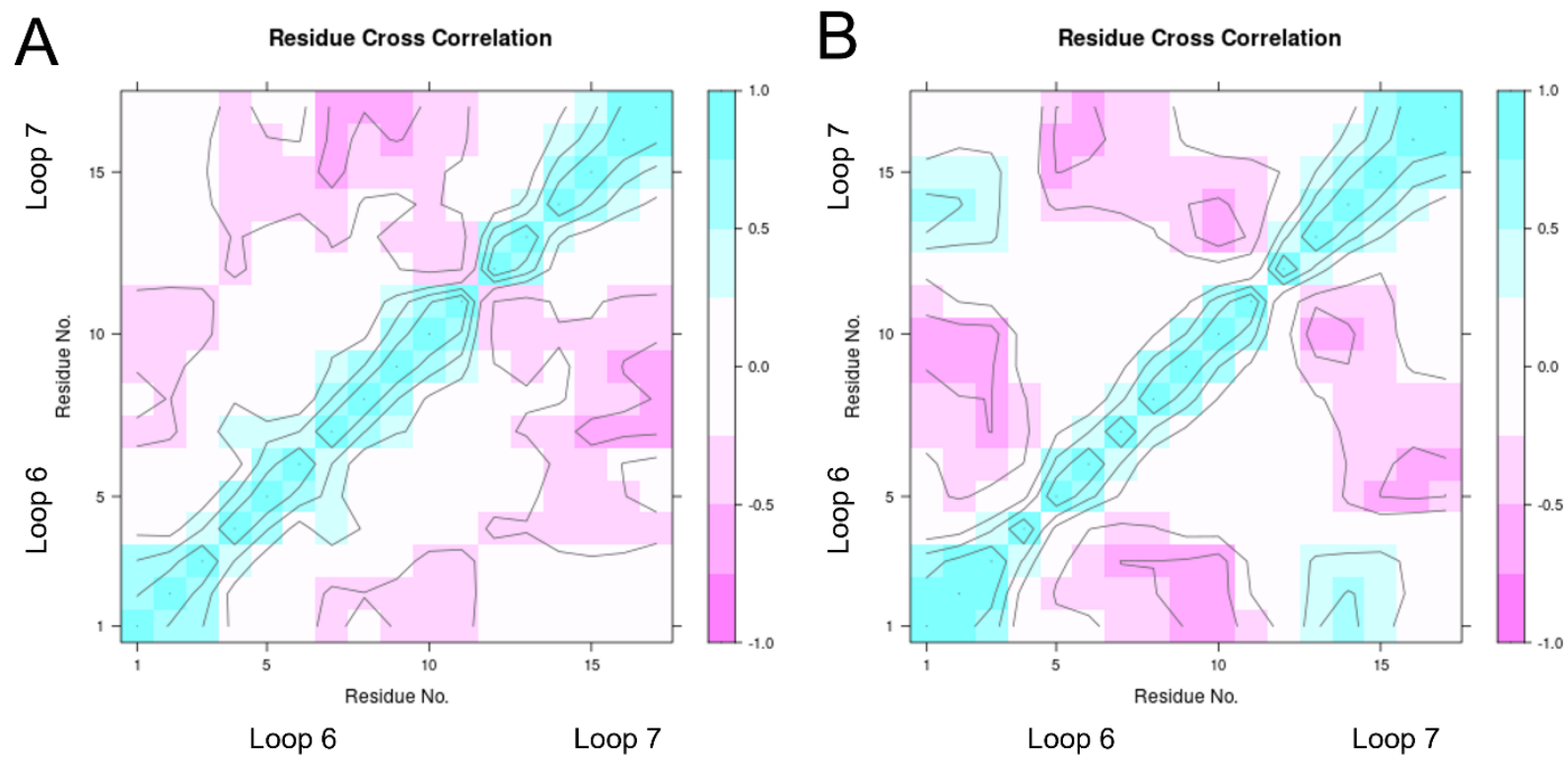

Fig. S7. Cross correlation between $\mathrm{C} \alpha$ atoms in loops 6 and 7 in the Mut 1 simulation in: (A) monomer A and (B) monomer B. Loop 6 corresponds to the first 11 amino acids and loop 7 to residues 12 to 17. The anti-correlation observed between the loops is in good agreement with the open-close transition observed in them. 


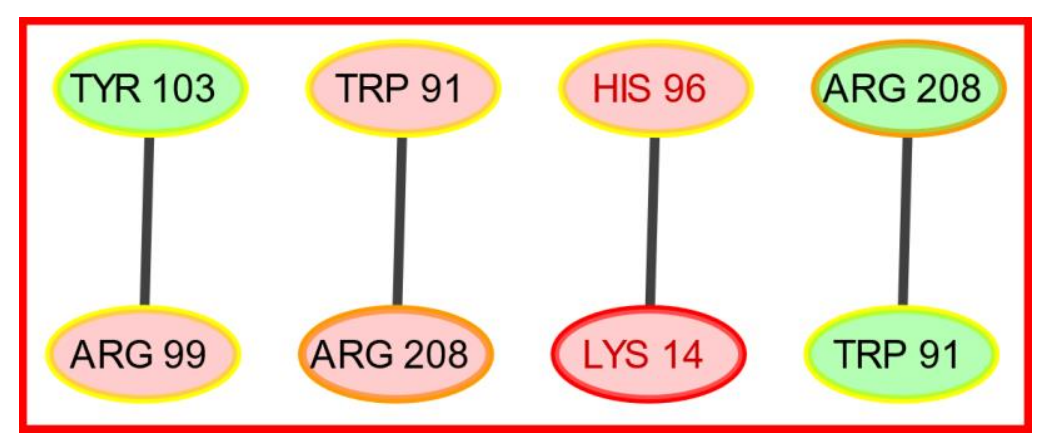

Fig. S8. Cation- $\pi$ interactions for TcTIM throughout the last $2 \mu$ s of the simulation. Amino acids in monomer A are shown in green and residues in monomer B in red. Each node is coloured according to the color scheme for regions in Fig. 1 of the main text. The catalytic residues are written with red text.
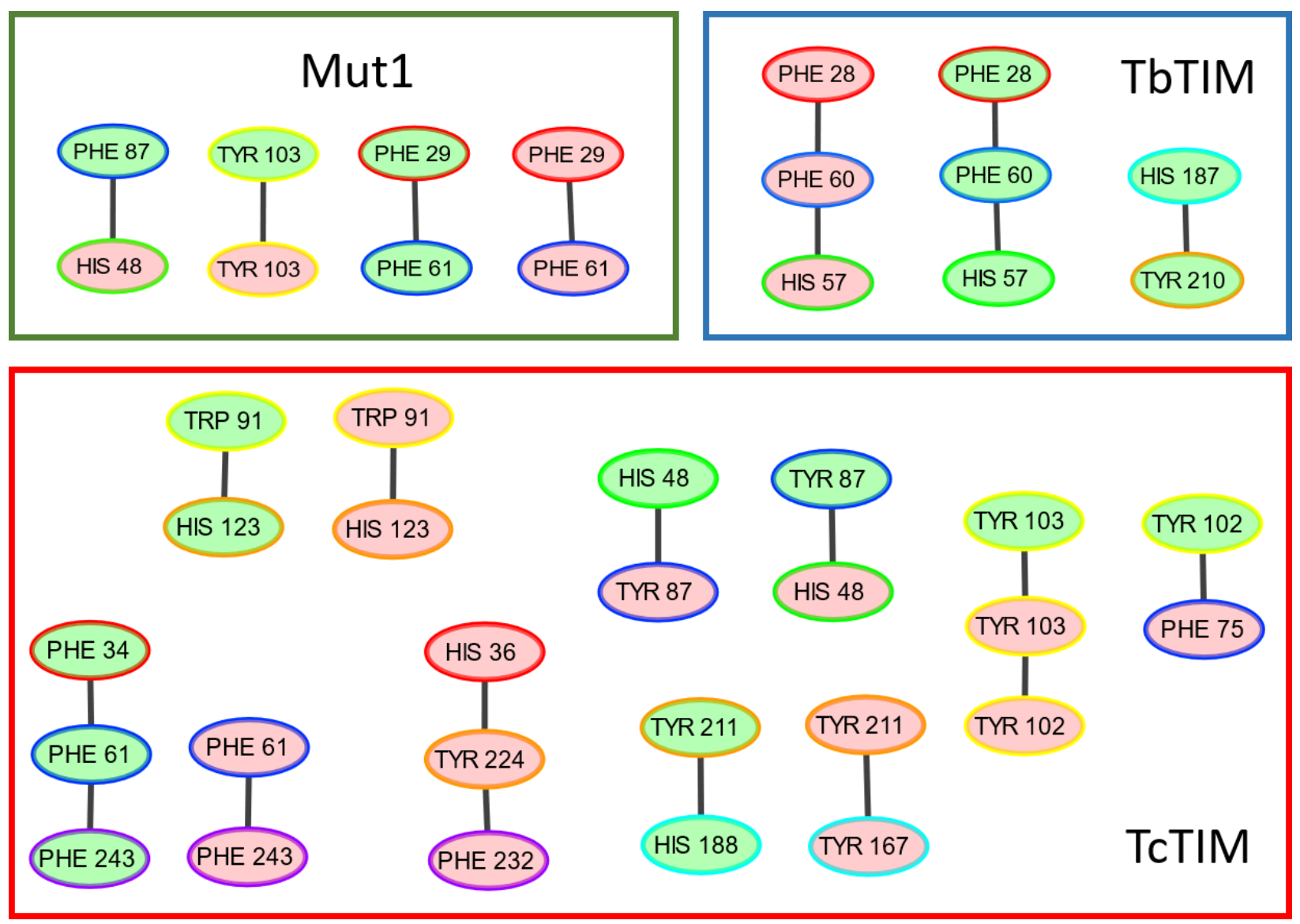

Fig. S9. $\pi-\pi$ interactions for TcTIM, TbTIM and Mut1 throughout the last $2 \mu$ s of the simulations. Amino acids in monomer $\mathrm{A}$ are shown in green and residues in monomer B in red. Each node is coloured according to the color scheme for regions in Fig. 1 of the main text. 

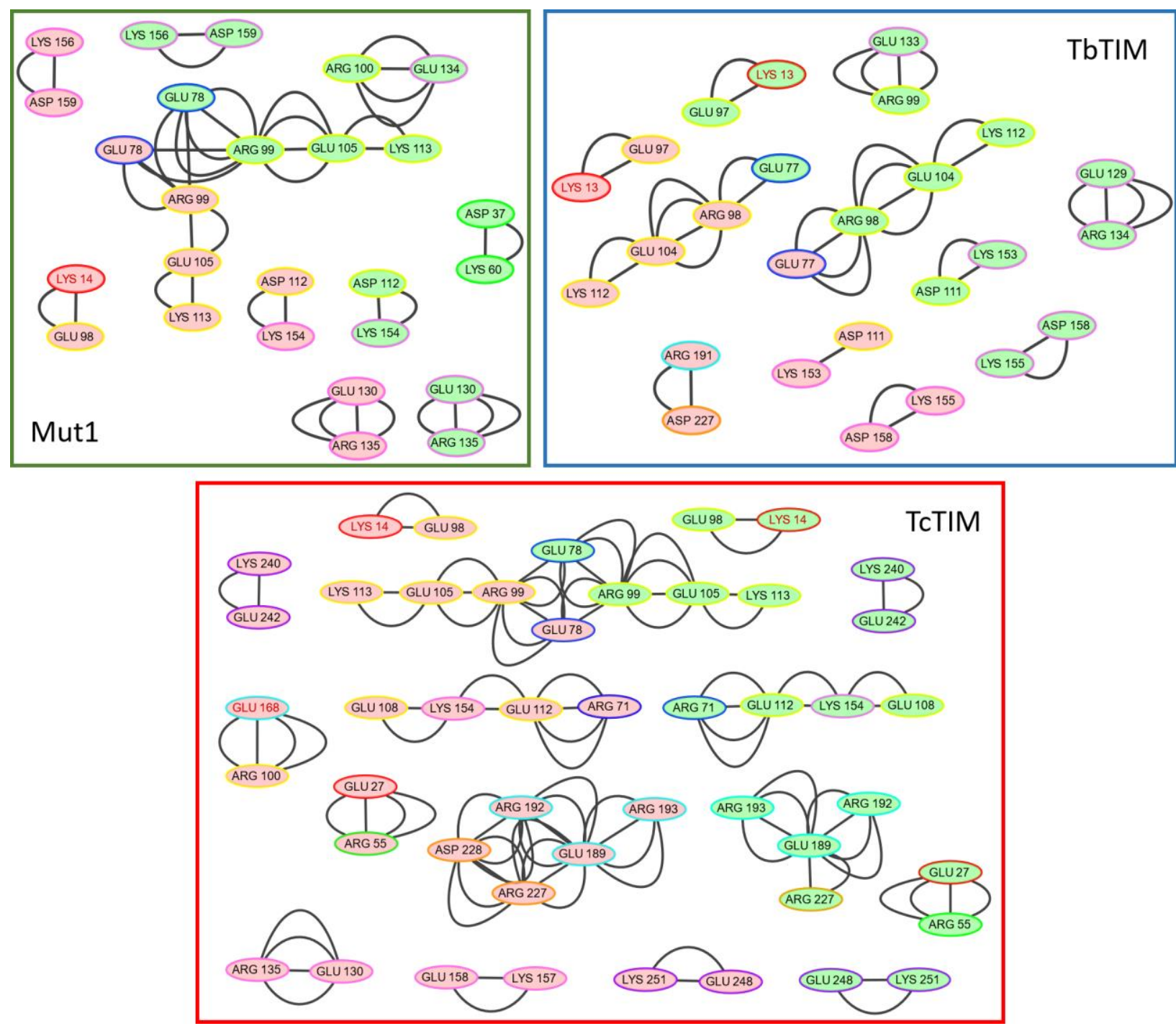

Fig. S10. Salt bridges for TcTIM, TbTIM and Mut1 throughout the last $2 \mu$ s of the simulations. TcTIM forms more salt bridges than the other two proteins. Amino acids in monomer A are shown in green and residues in monomer B in red. Each node is coloured according to the color scheme for regions in Fig. 1 of the main text. The catalytic residues are written with red text. 


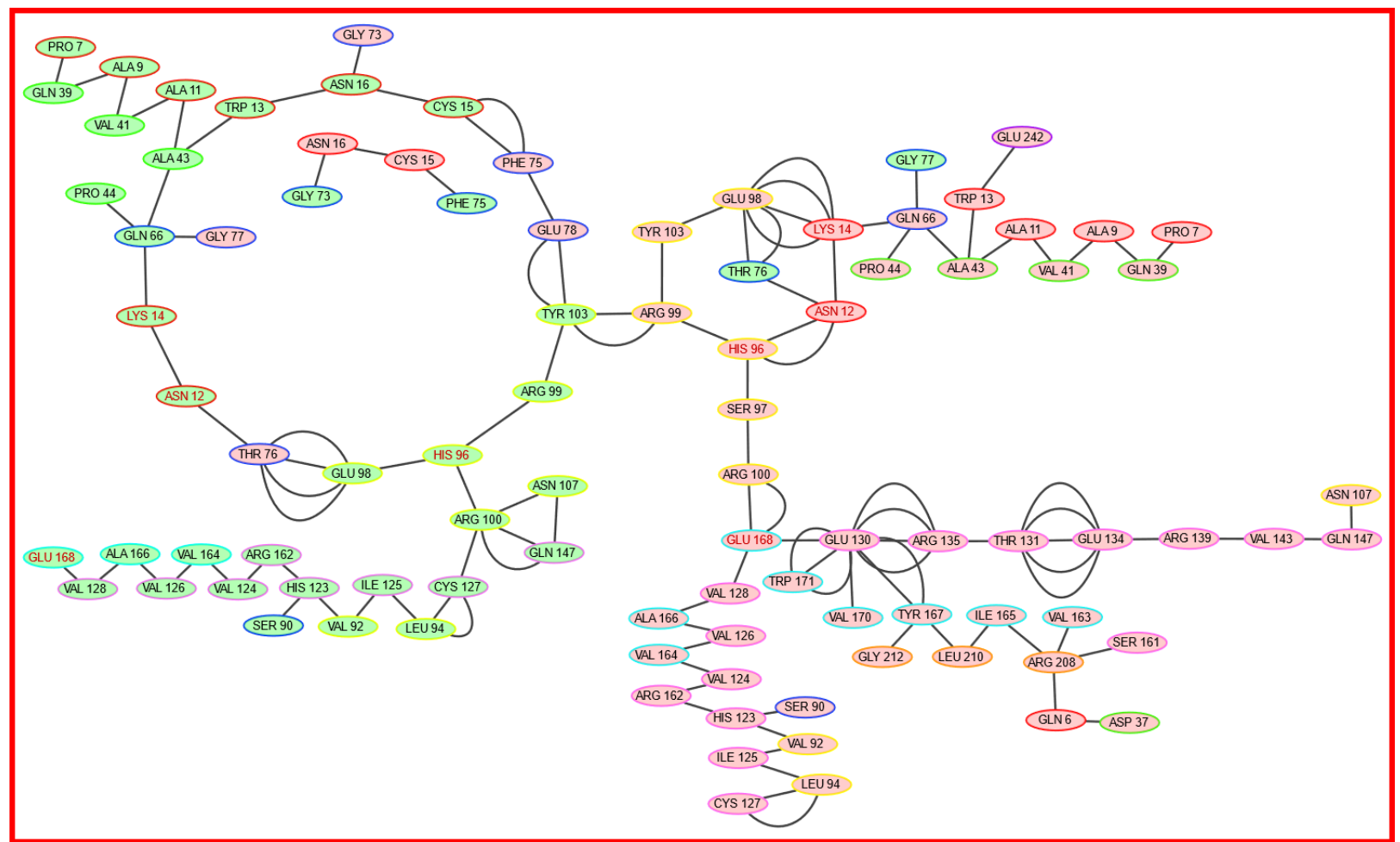

Fig. S11. Main hydrogen bond network in TcTIM throughout the last $2 \mu$ s of the simulation. Each node is coloured according to the color scheme for regions in Fig. 1 of the main text. The catalytic residues are written with red text.

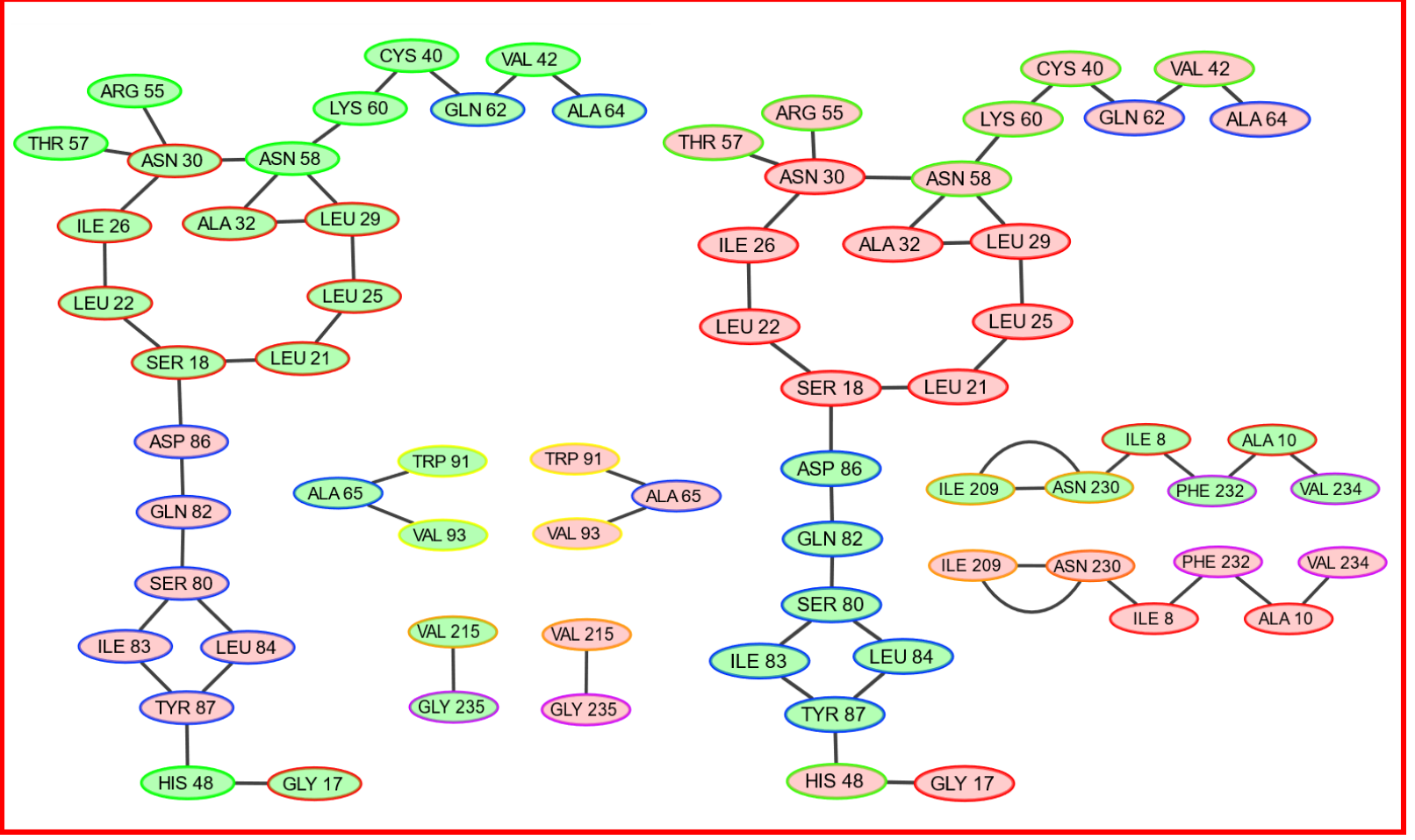

Fig. S12. Hydrogen bonds in TcTIM throughout the last $2 \mu$ s of the simulation. These hydrogen bonds are found in both monomers. Amino acids in monomer A are shown in green and residues in monomer B in red. Each node is coloured according to the color scheme for regions in Fig. 1 of the main text. 


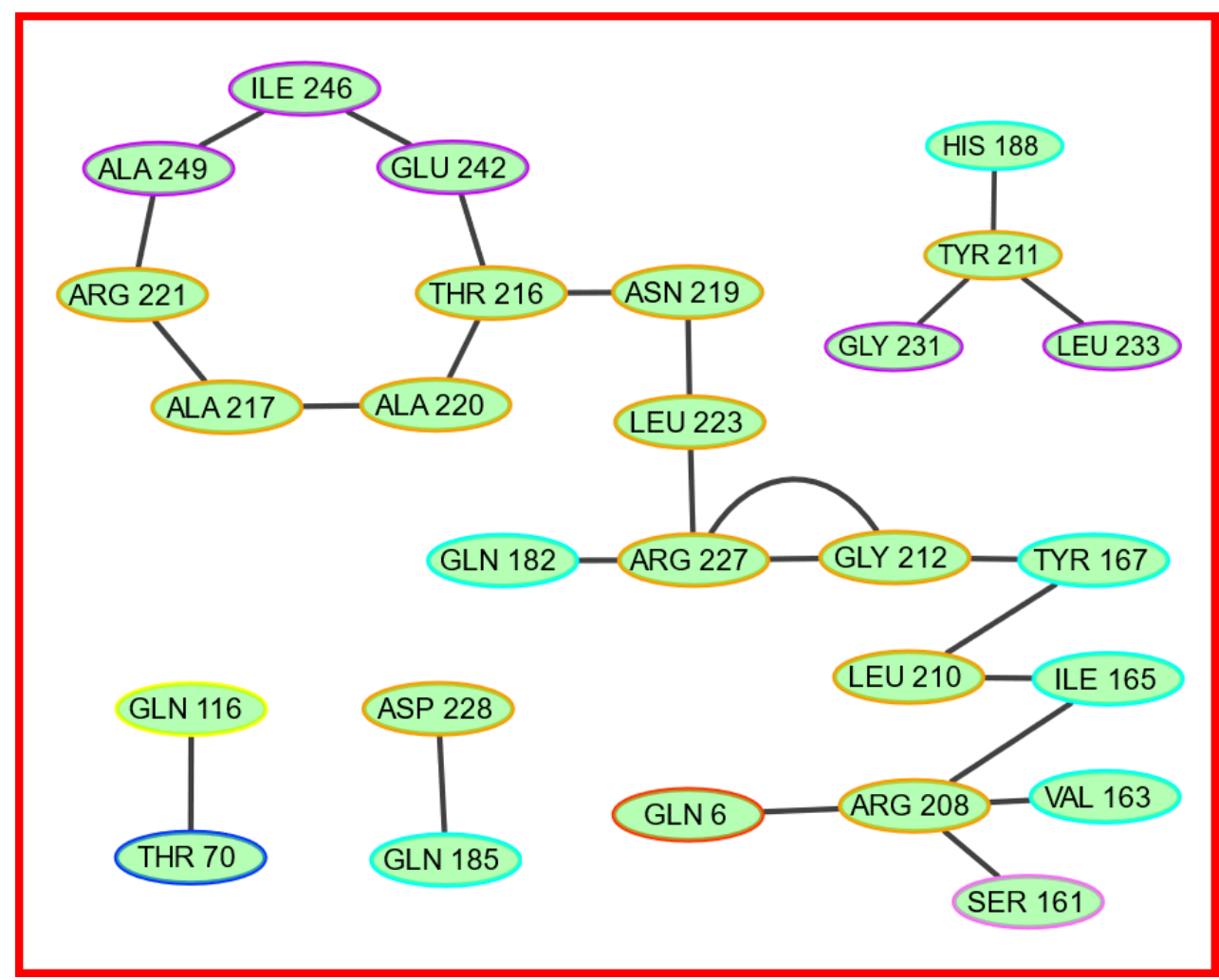

Fig. S13. Hydrogen bonds in monomer A throughout the last $2 \mu$ s of the TcTIM simulation. Each node is coloured according to the color scheme for regions in Fig. 1 of the main text.

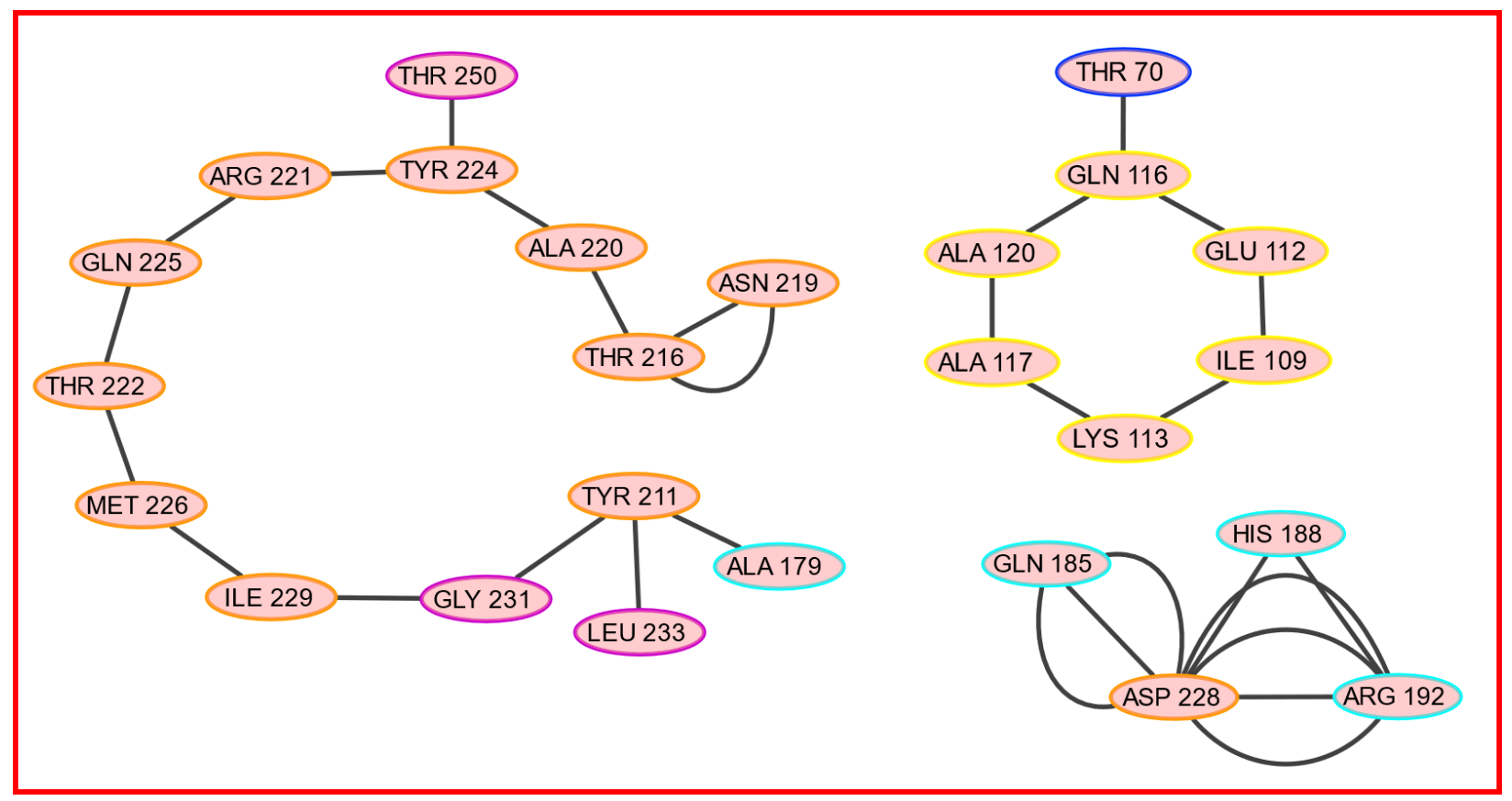

Fig. S14. Hydrogen bonds in monomer B throughout the last $2 \mu$ s of the TcTIM simulation. Each node is coloured according to the color scheme for regions in Fig. 1 of the main text. 

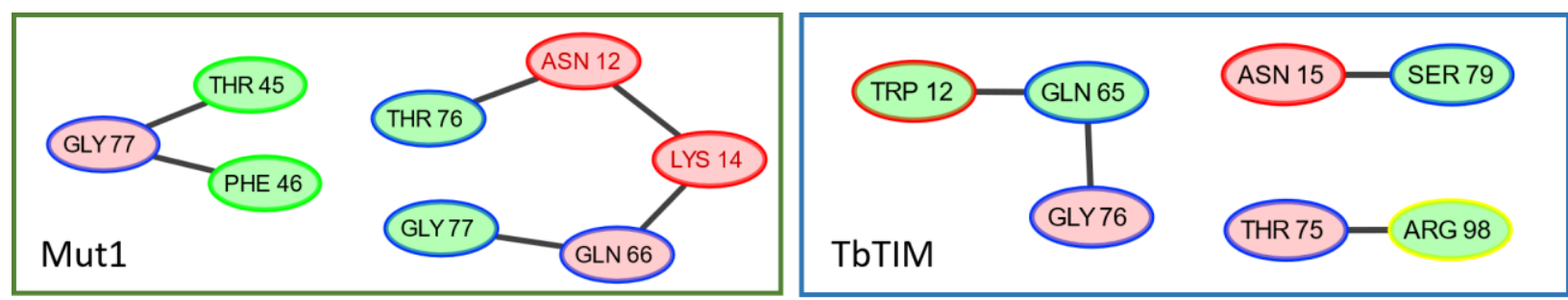

Fig. S15. Hydrogen bonds involving amino acids at the interface between monomers in Mut1 and TbTIM throughout the last $2 \mu \mathrm{s}$ of the simulations. Amino acids in monomer A are shown in green and residues in monomer B in red. Each node is coloured according to the color scheme for regions in Fig. 1 of the main text. The catalytic residues are written with red text.

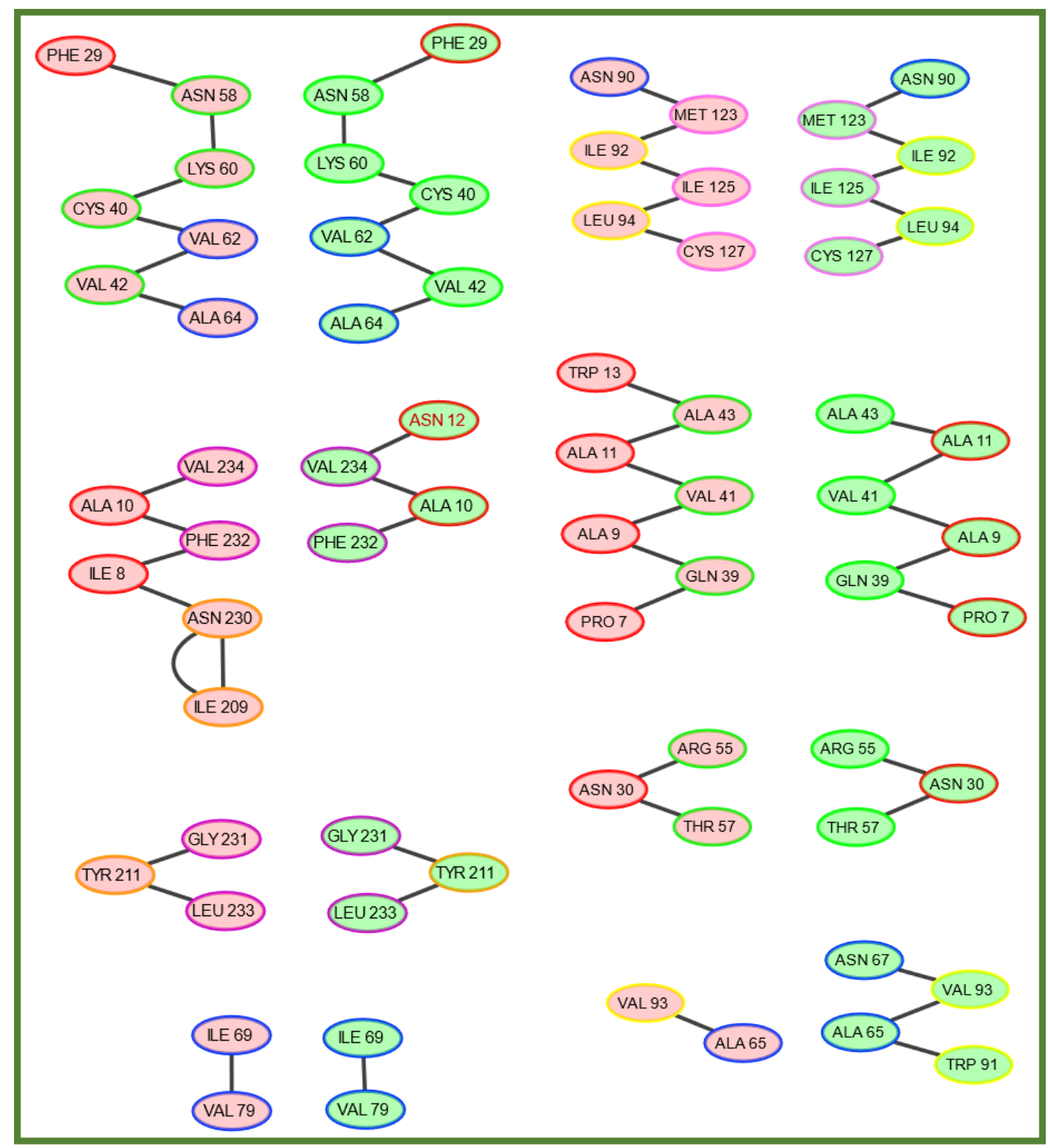

Fig. S16. Hydrogen bonds in Mut 1 throughout the last $2 \mu$ s of the simulation. These hydrogen bonds are found in both monomers. Amino acids in monomer A are shown in green and residues in monomer B in red. Each node is coloured according to the color scheme for regions in Fig. 1 of the main text. The catalytic residue is written with red text. 


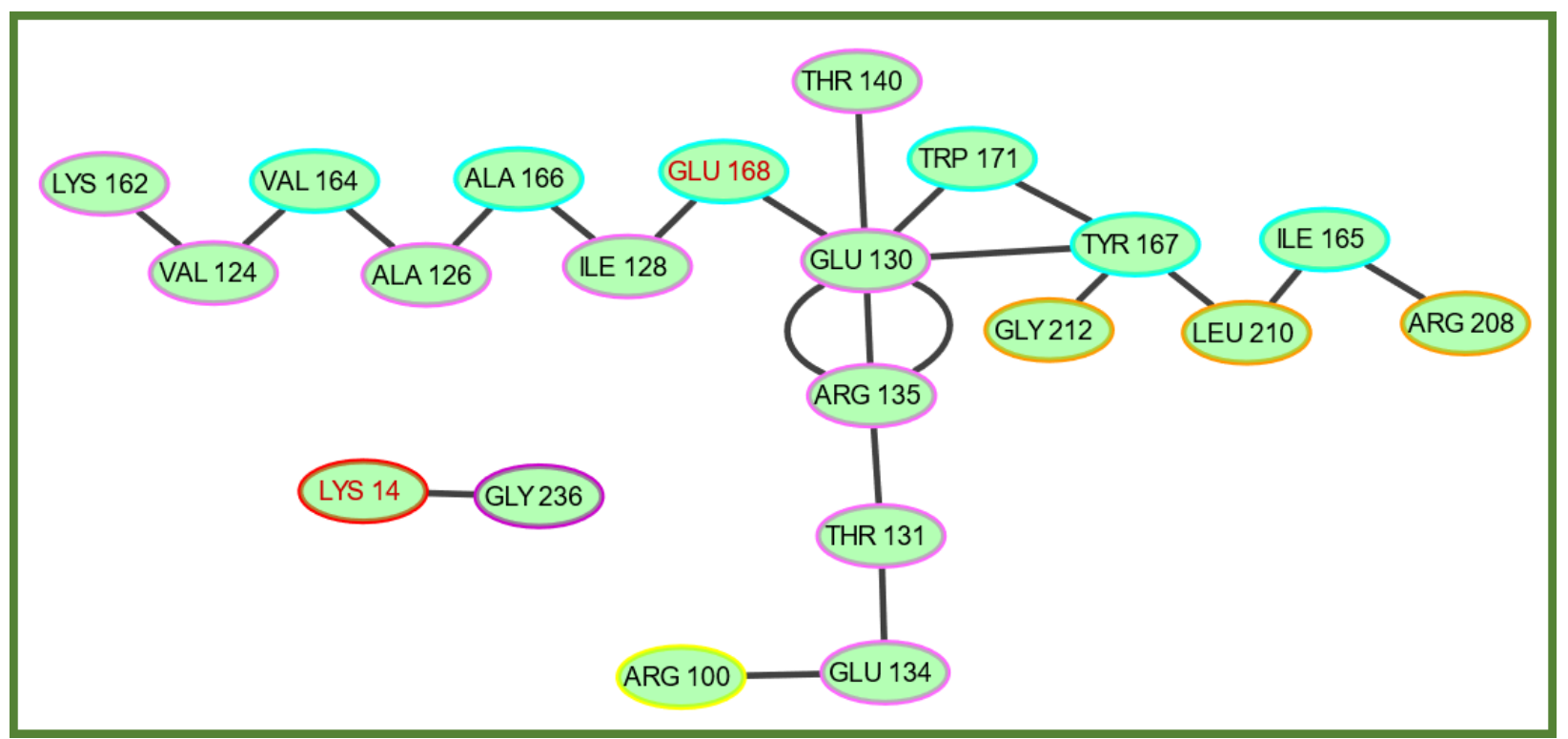

Fig. S17. Hydrogen bonds in monomer A throughout the last $2 \mu$ s of the Mut 1 simulation. Each node is coloured according to the color scheme for regions in Fig. 1 of the main text. The catalytic residues are written with red text.

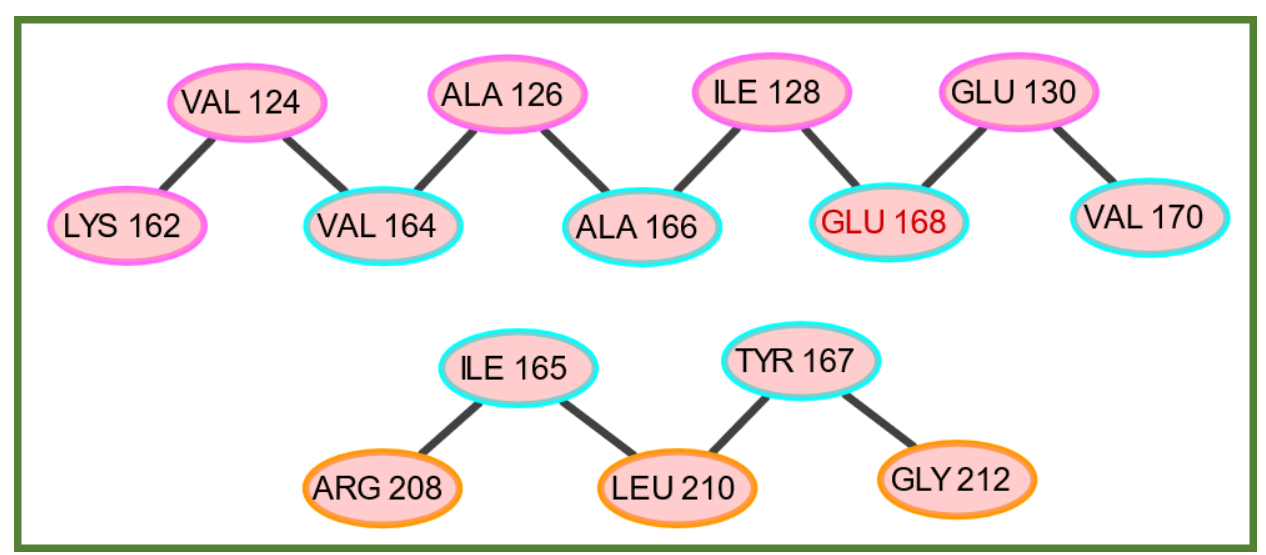

Fig. S18. Hydrogen bonds in monomer B throughout the last $2 \mu$ s of the Mut 1 simulation. Each node is coloured according to the color scheme for regions in Fig. 1 of the main text. The catalytic residue is written with red text. 

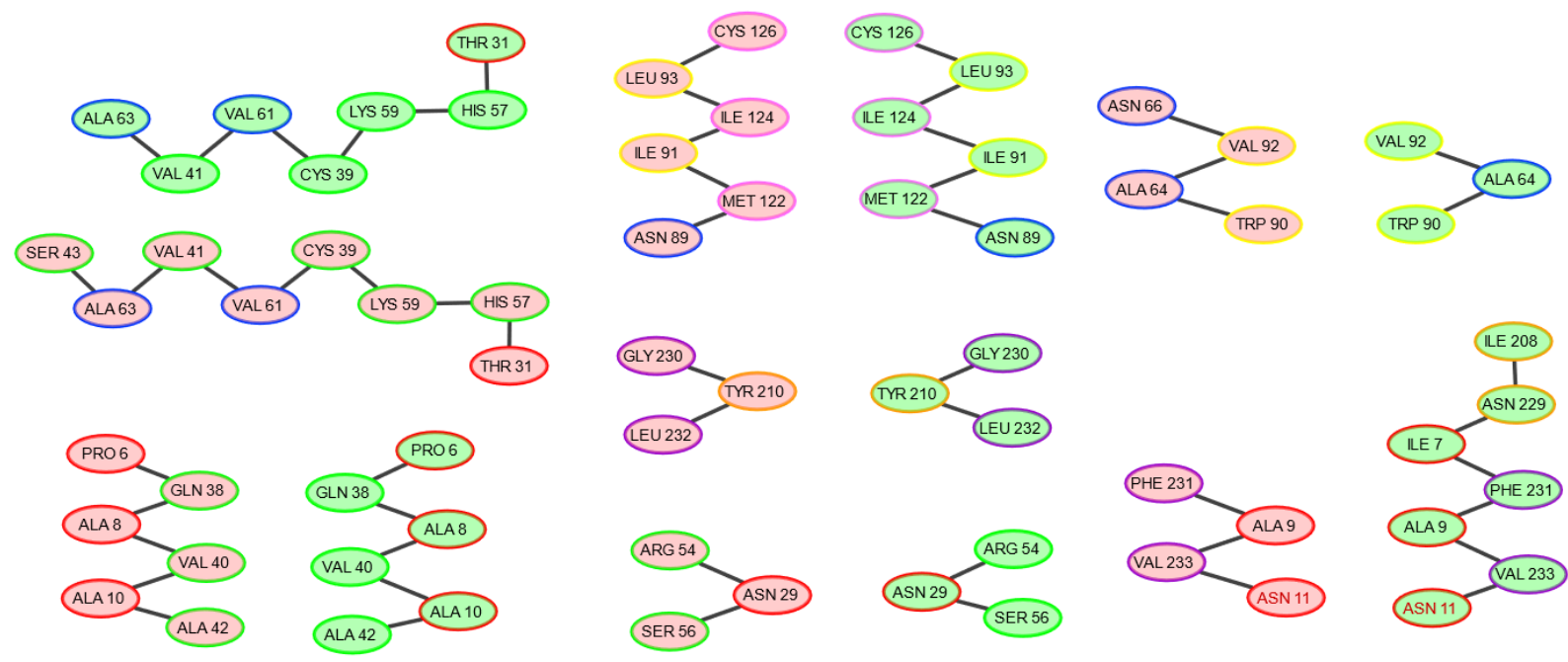

Fig. S19. Hydrogen bonds in TbTIM throughout the last $2 \mu$ s of the simulation. These hydrogen bonds are found in both monomers. Amino acids in monomer A are shown in green and residues in monomer B in red. Each node is coloured according to the color scheme for regions in Fig. 1 of the main text. The catalytic residues are written with red text.

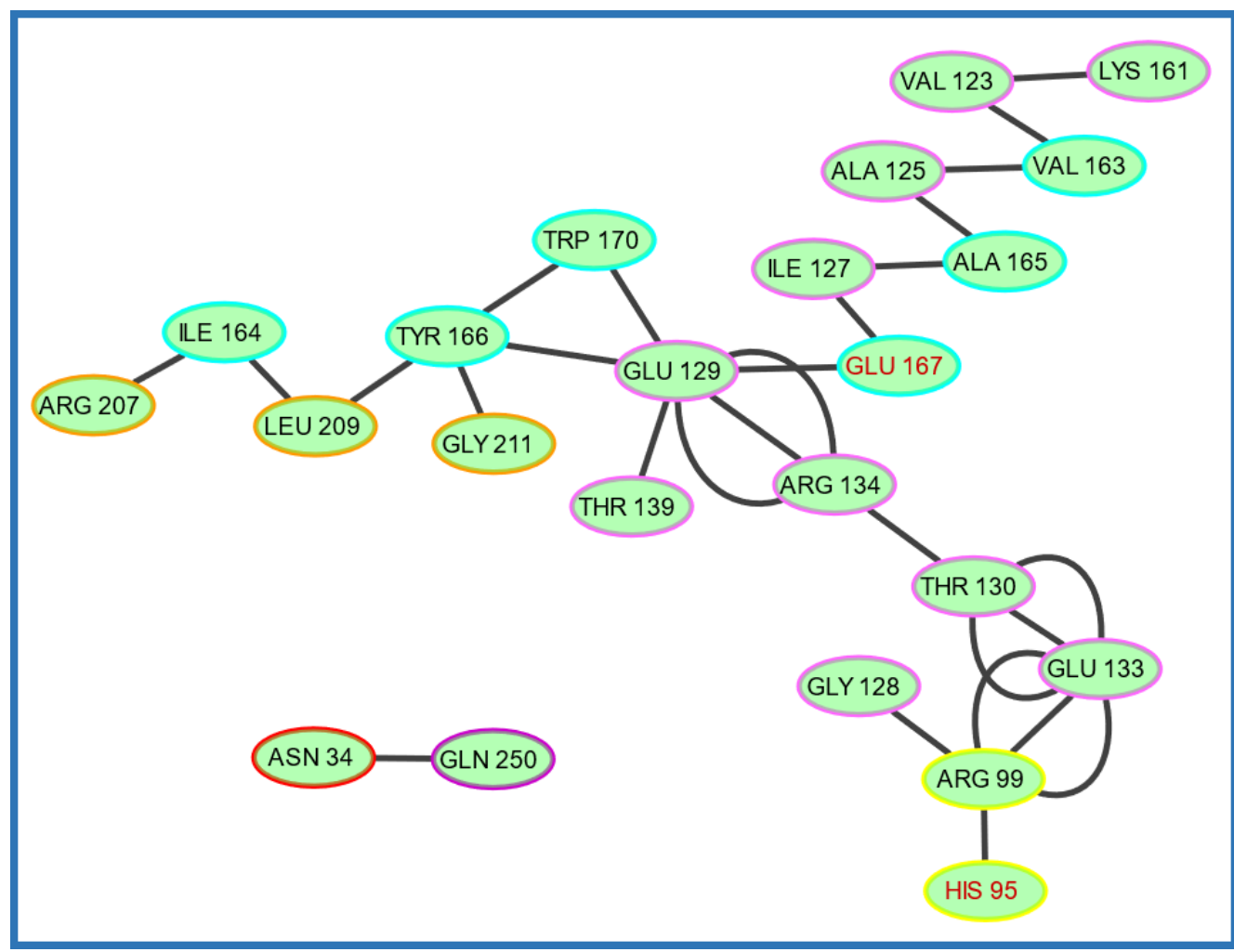

Fig. S20. Hydrogen bonds in monomer A throughout the last $2 \mu$ s of the TbTIM simulation. Each node is coloured according to the color scheme for regions in Fig. 1 of the main text. The catalytic residue is written with red text. 


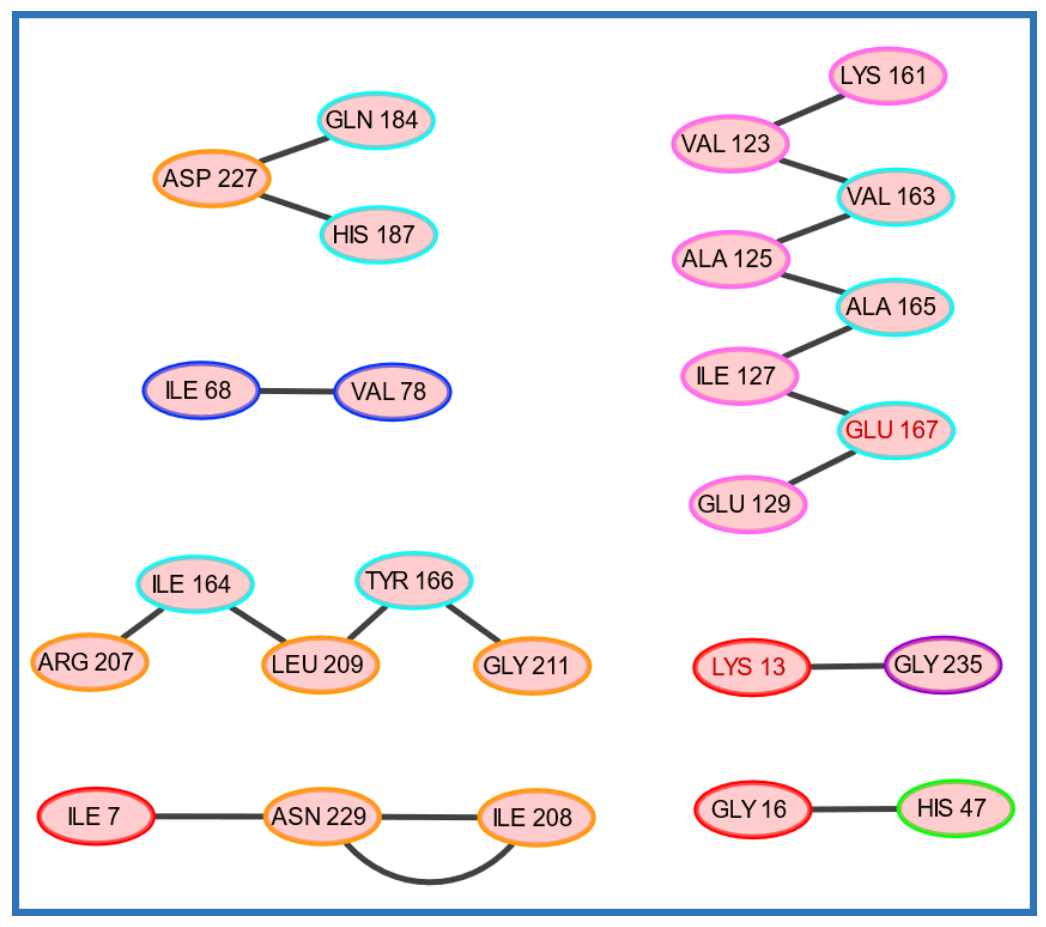

Fig. S21. Hydrogen bonds in monomer B throughout the last $2 \mu$ s of the TbTIM simulation. Each node is coloured according to the color scheme for regions in Fig. 1 of the main text. The catalytic residues are written with red text.
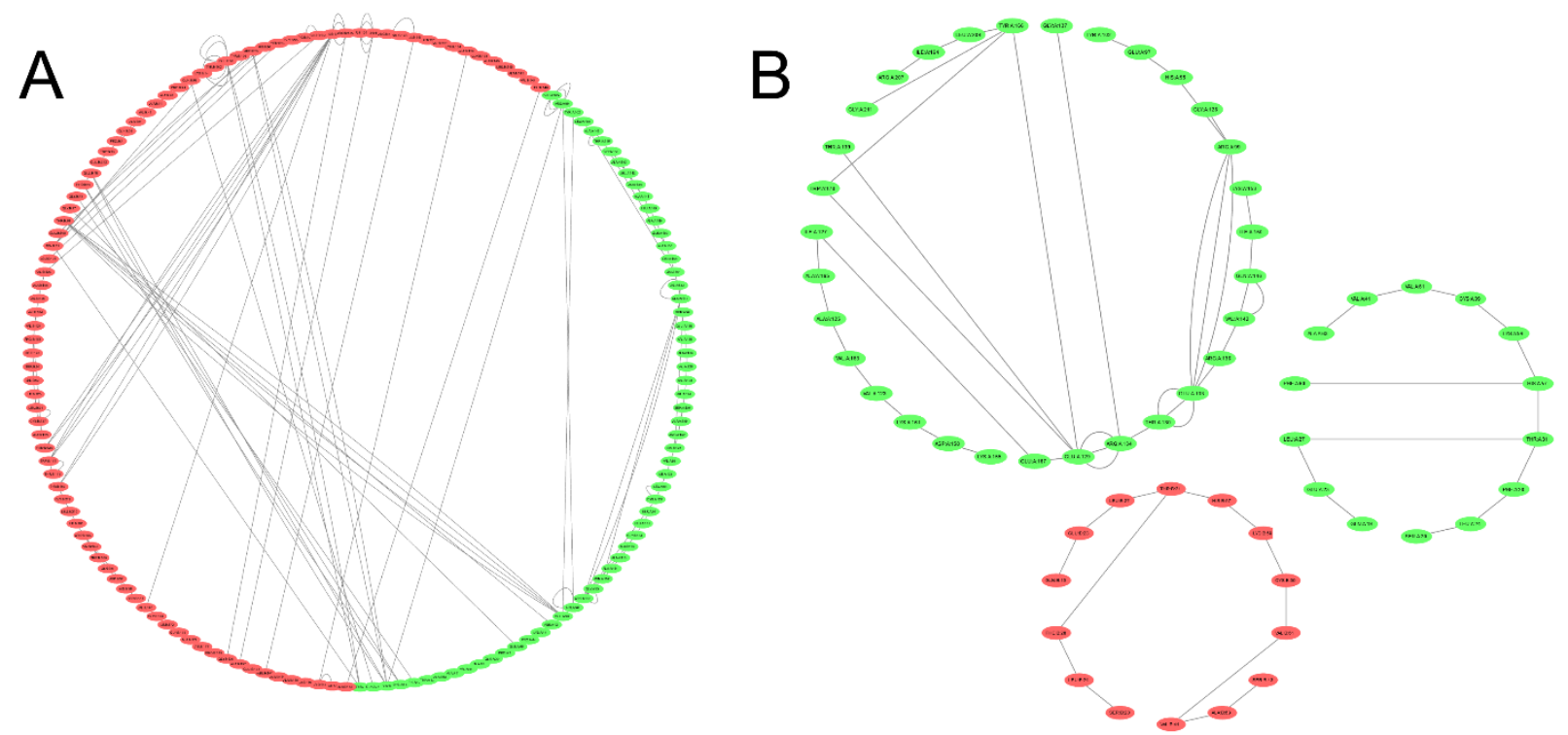

Fig. S22. Main hydrogen bond networks throughout the last $2 \mu$ s for A) TcTIM and B) TbTIM. Amino acids in monomer A are shown in green and residues in monomer B in red. Hydrogen bonds in TcTIM connect amino acids in a network that involves many interactions between monomers and extends throughout the whole protein, in contrast with TbTIM, whose networks are contained within each monomer and involve fewer residues. 
A
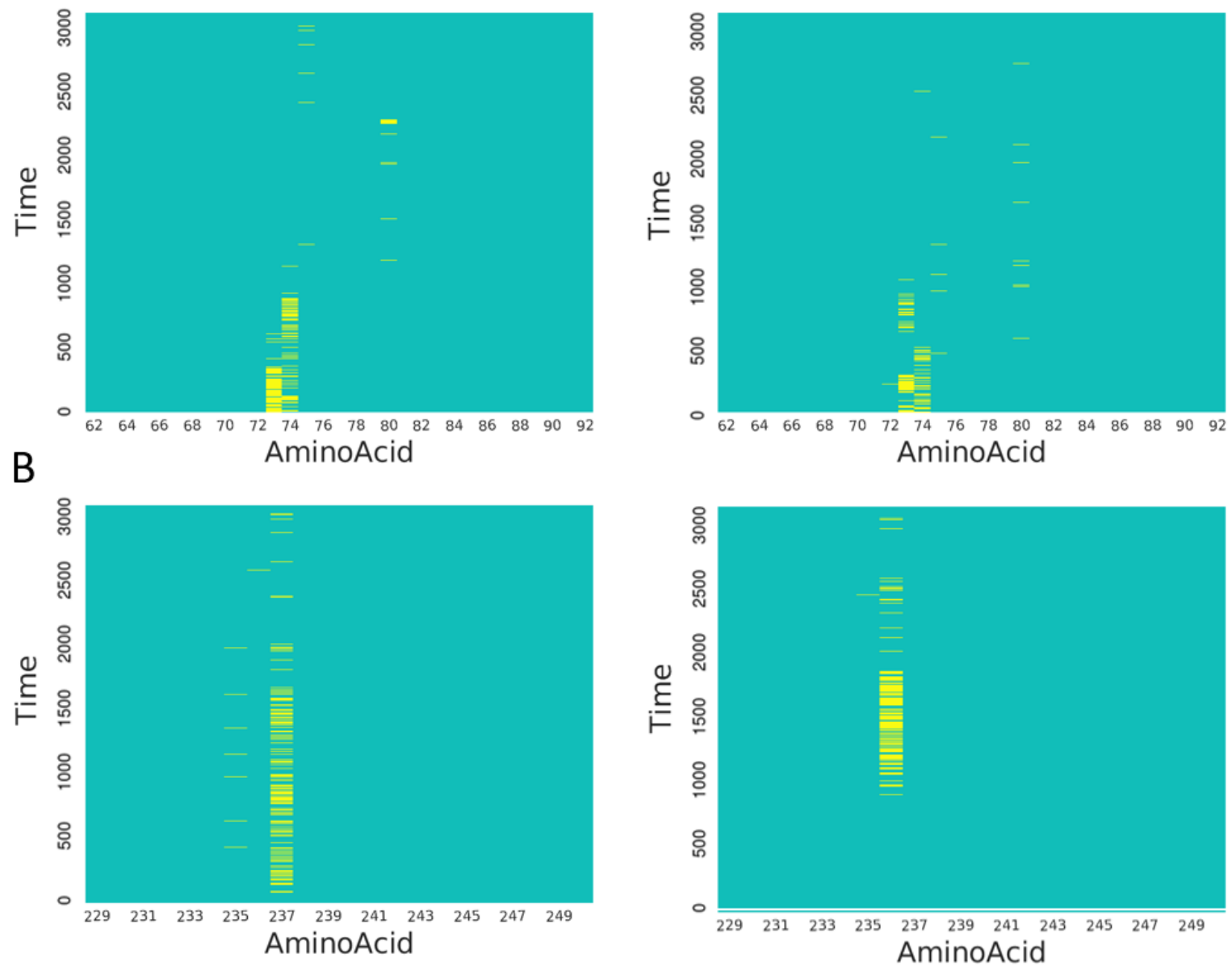

C
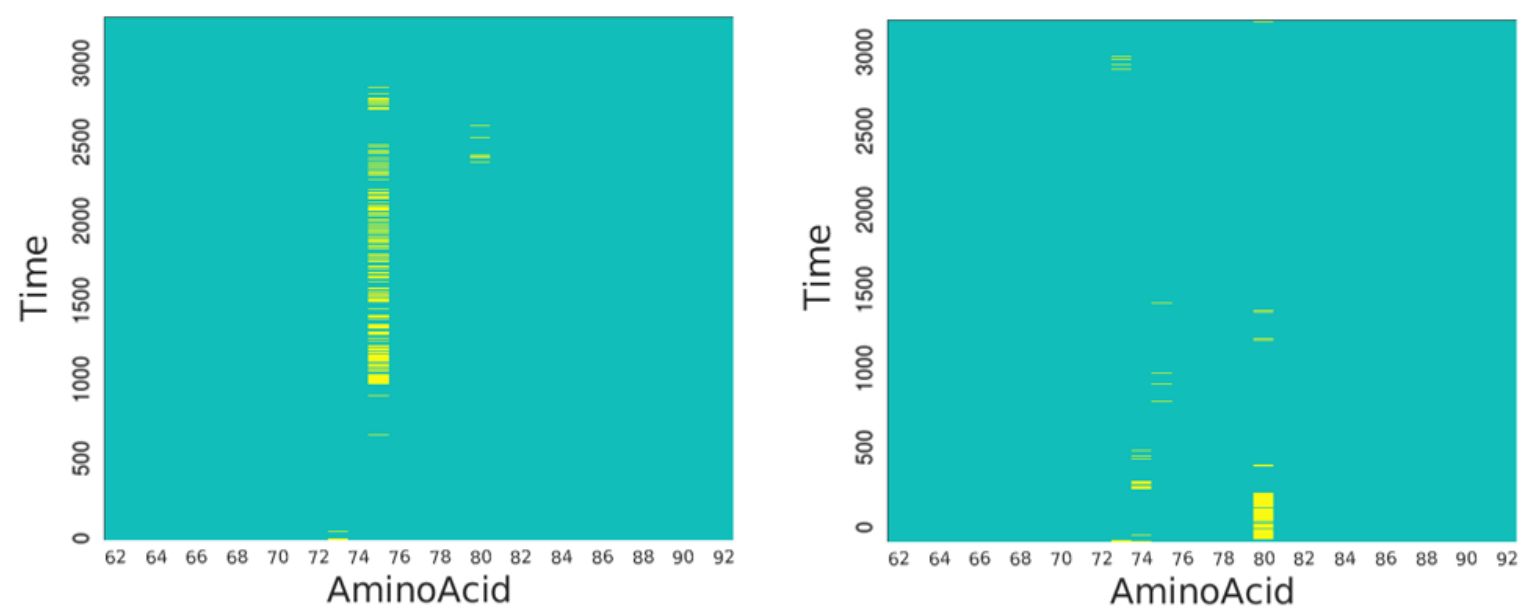

Fig. S23. Hydrogen bonds in Cys 14/15 monomer A (left) and monomer B (right) in: A) TcTIM, B) TbTIM and C) Mut1. This cysteine forms hydrogen bonds with region 3 of the other monomer in TcTIM and Mut 1 and forms hydrogen bonds in region 8 of the same monomer in TbTIM. 
Table S1. Summary of the key findings in all three simulations. Red: catalytic amino acids.

\begin{tabular}{|c|c|c|c|c|c|}
\hline & TcTIM & TbTIM & Mut1 & Impact & Experiments \\
\hline RMSF & $\begin{array}{l}\text { Peaks in } \\
\text { loops } 5 \& 6\end{array}$ & $\begin{array}{c}\text { Peaks in } \\
\text { loops } 5 \& 6\end{array}$ & $\begin{array}{c}\text { No peak in } \\
\text { loop5, small } \\
\text { peak in loop } \\
6\end{array}$ & $\begin{array}{l}\text { Mut1 may have } \\
\text { lower catalytic } \\
\text { activity }\end{array}$ & - \\
\hline $\begin{array}{l}\text { Contacts at } \\
\text { interface } \\
\text { between } \\
\text { monomers }\end{array}$ & 119 & 89 & 116 & $\begin{array}{l}\text { TcTIM's thermal } \\
\text { stability is higher } \\
\text { than TbTIM's }\end{array}$ & $\begin{array}{l}\text { In agreement } \\
\text { with Ref. (22) }\end{array}$ \\
\hline Cation-т & $\begin{array}{c}4 \\
\text { interactions }\end{array}$ & - & - & - & - \\
\hline $\begin{array}{l}\text { Glu } 168- \\
\text { Arg } 100\end{array}$ & $\begin{array}{c}\text { Salt bridge }+ \\
\text { Hbond }\end{array}$ & - & - & - & - \\
\hline $\begin{array}{l}\text { Lys } 14 \text { - } \\
\text { Glu98 }\end{array}$ & Salt bridge & Salt bridge & Salt bridge & $\begin{array}{l}\text { Conserved } \\
\text { interaction }\end{array}$ & $\begin{array}{c}\text { In agreement } \\
\text { with Refs. } \\
(30,31)\end{array}$ \\
\hline $\begin{array}{c}\text { Asn12- } \\
\text { Val234 }\end{array}$ & - & Hbond & Hbond & - & - \\
\hline $\begin{array}{l}\text { Lys14- } \\
\text { Gly236 }\end{array}$ & - & Hbond & Hbond & - & - \\
\hline $\begin{array}{l}\text { Glu168- } \\
\text { Val128 }\end{array}$ & Hbond & Hbond & Hbond & - & - \\
\hline $\begin{array}{l}\text { Glu168- } \\
\text { Glu130 }\end{array}$ & Hbond & Hbond & Hbond & - & - \\
\hline $\begin{array}{l}\text { Arg192 - } \\
\text { Asp228 }\end{array}$ & Salt bridge & Salt bridge & - & $\begin{array}{l}\text { Conserved, } \\
\text { important for } \\
\text { efficient folding }\end{array}$ & $\begin{array}{l}\text { In agreement } \\
\text { with Ref. (64) }\end{array}$ \\
\hline
\end{tabular}


A

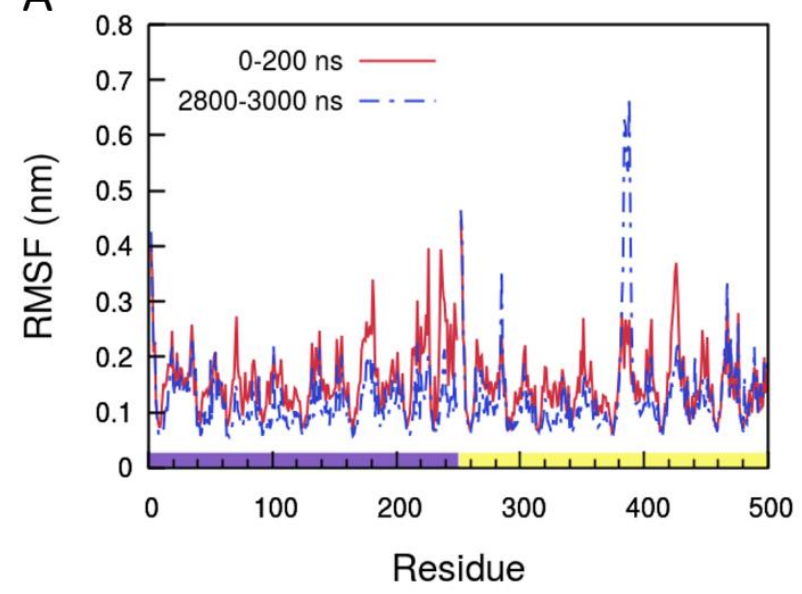

B

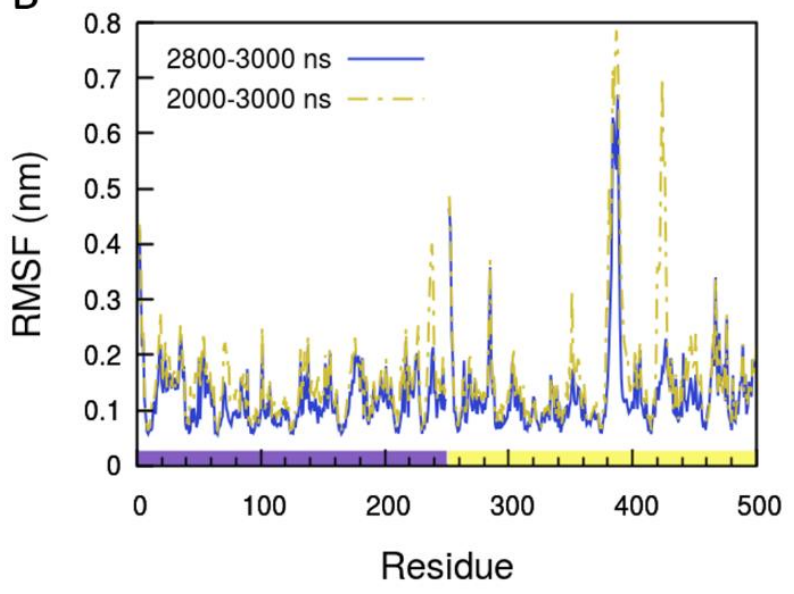

Fig. S24. Changes over time in the root mean square fluctuation (RMSF) of the TbTIM simulation. RMSF of the first $200 \mathrm{~ns}$ of the simulation vs the last $200 \mathrm{~ns}$ (A), and RMSF of the last microsecond of the trajectory vs the last $200 \mathrm{~ns}$ (B). The color bar at the bottom of figure B distinguishes the residues in monomer A (purple) from those in monomer $\mathrm{B}$ (yellow). There are no significant peaks in the RMSF of the beginning of the trajectory. The last $200 \mathrm{~ns}$ of the simulation failed to capture the peak at loop 6 monomer B.

A

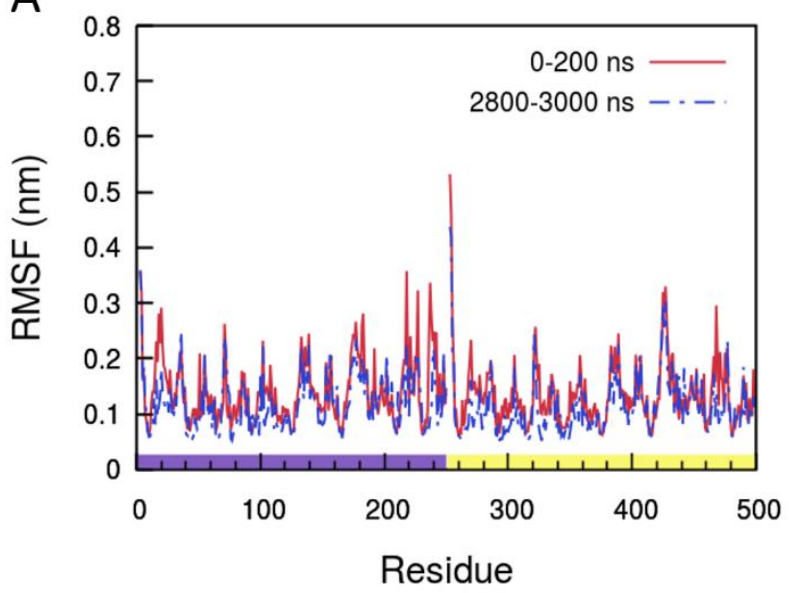

B

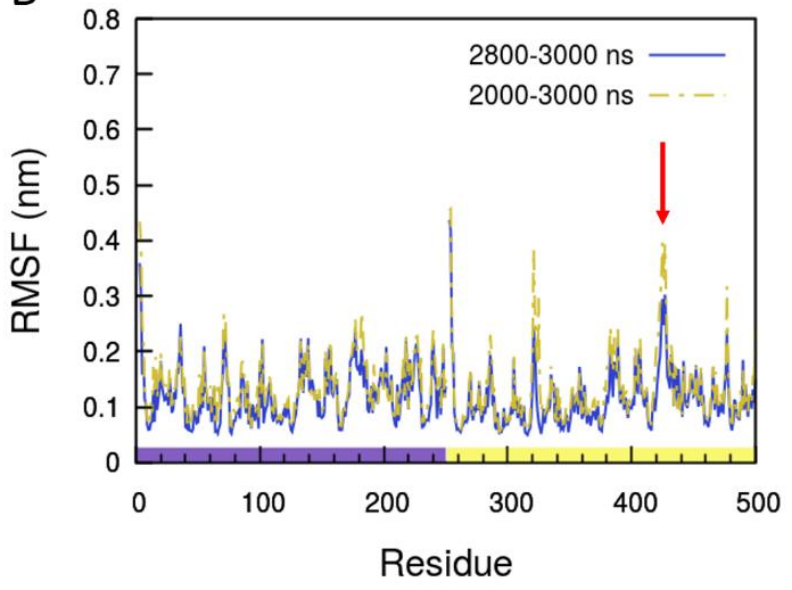

Fig. S25. Changes over time in the root mean square fluctuation (RMSF) of the Mut1 simulation. RMSF of the first $200 \mathrm{~ns}$ of the simulation vs the last $200 \mathrm{~ns}$ (A), and RMSF of the last microsecond of the trajectory vs the last $200 \mathrm{~ns}$ (B). The color bar at the bottom of figure B distinguishes the residues in monomer A (purple) from those in monomer $\mathrm{B}$ (yellow). Fluctuations at the minor peaks decreased with time and the peak at loop 6 monomer B (red arrow) increased. 


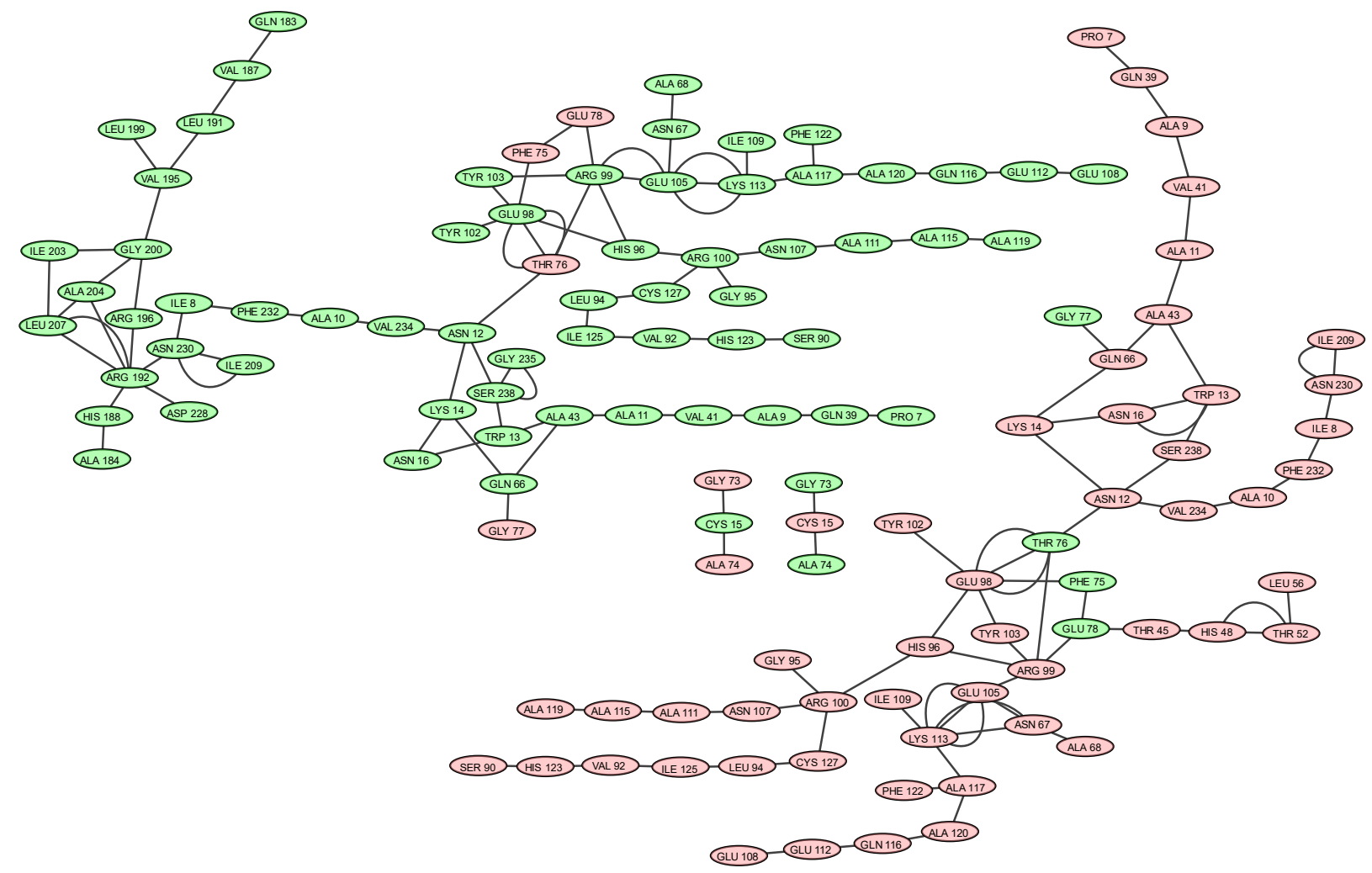

Fig. S26. Main hydrogen bond networks for TcTIM in the most populated cluster of the first 500 ns of the simulation. There is a different network for each monomer. Amino acids in monomer A are shown in green and residues in monomer $\mathrm{B}$ in red. 


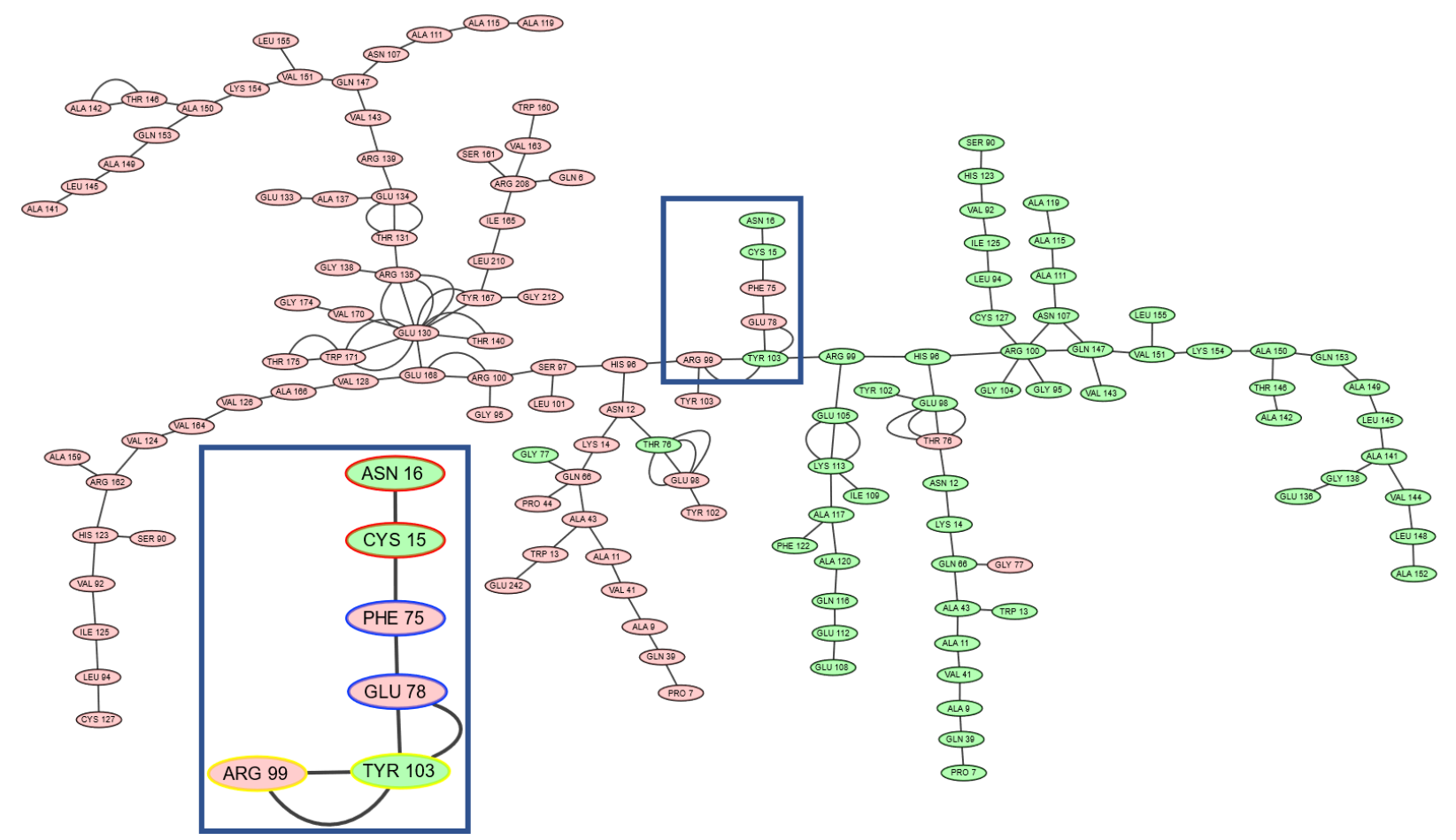

Fig. S27. Hydrogen bond networks for TcTIM in the most populated cluster of the last 500 ns of the simulation. The residues in both monomers are connected through a single network of hydrogen bonds. Highlighted in blue are the residues at the interface of the hydrogen bond network (Fig. 11 in the main text). Amino acids in monomer $\mathrm{A}$ are shown in green and residues in monomer $\mathrm{B}$ in red. 